\title{
Tuberous sclerosis complex: an opportunistic diagnosis
}

\author{
Liliana Torres, Teresa Martins Mendes, Diana Pereira Anjos, Mari Mesquita \\ Centro Hospitalar do Tâmega e Sousa. Portugal
}

\begin{abstract}
Tuberous sclerosis complex (TSC) is a rare genetic multisystem disease that involves brain, skin, kidneys, eyes and lungs. It typically presents during childhood with facial adenomas, seizures and mental retardation and its diagnosis is mainly clinical. We report a case of 68-year-old man that sought medical care due to a transient ischemic stroke. The past clinical history and physical observation showed symptoms and signs of TSC. An extend imagiological study allowed for a complete assessment of organ involvement. A delayed diagnosis places adults at increased risk for morbidity and mortality and therefore, clinicians must be familiar with the full spectrum of TSC-associated diagnostic features.
\end{abstract}

Keywords: Angiofibromas. Tubers.

\section{Introduction}

Tuberous sclerosis complex (TSC) is an neurocutaneous disorder affecting cell development and maturation and is characterized by pleomorphic features that can affect every organ system. The classic TSC diagnostic triad of seizures, intellectual disability and facial angiofibromas (Vogt triad) occurs in less than one--third of patients with TSC. Though its treatment is only symptomatic, an early diagnosis is crucial to prevent long term organ system damage ${ }^{1}$.

\section{Case report}

A67--year--old man presented in the emergency room with a transient ischemic stroke ABCD2 score 4. No other neurological or systemic symptoms were present. On observation, he had multiple pebble--like pink tubers on his facial mask below the lower rim of the eyes and extending to the cheeks and composure of his mouth, as well as just below his lower lip and above his chin (Figure 1). On his lower back, he had a tuberous growth with a smooth surface and dimensions of approximately $10 \mathrm{~mm}$ of diameter and 2 to $3 \mathrm{~mm}$ in height (Figure 2). His finger and toe nail beds were normal. His past medical history included mental retardation with learning disability that made him drop out of school at 11 years old. As a single man without offspring, he lived with his sister after a psychological evaluation, at age 40 , showed him unfit to work due to his childish behavior. His father had had epilepsy but there was no other relevant family history. Haematological and biochemical profile had no alterations. Computed tomography (CT) scan of the brain revealed subependymal and supratentorial nodules. Magnetic resonance imaging showed bilateral fronto--parieto-occipital tubers (Figure 3). His electroencephalogram was normal. Thoracic, abdominal and pelvic CT scans were normal. An ophtalmologic evaluation revealed 2 retinal hamartomas on his right eye. No areas of retinal hypopigmentation were present. A cardiac resonance showed a rhabdomyoma in the left ventricle without compromise of its function. He was dismissed with the diagnosis of Tuberous Sclerosis Complex with brain, eyes and cardiac involvement. The patient maintains annual surveillance in our hospital.
Fig. 1. Facial angiofibromas

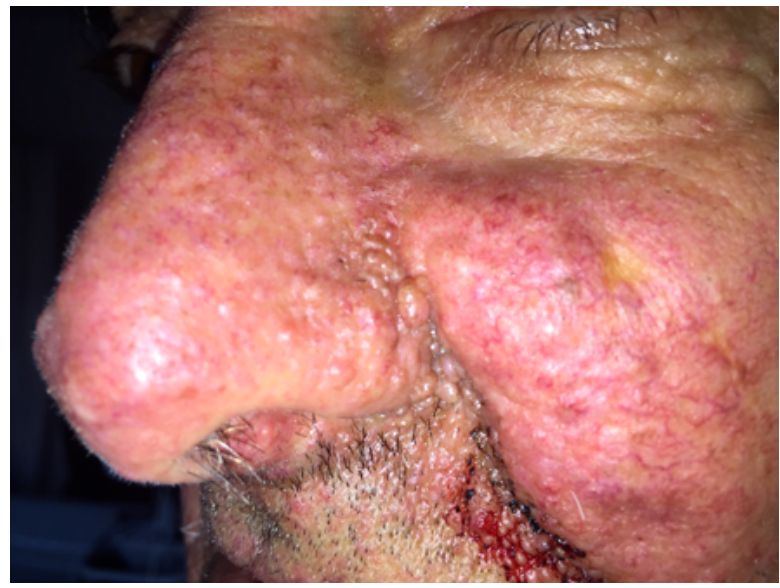

Fig. 2. Tuberous growth in the lumbar región

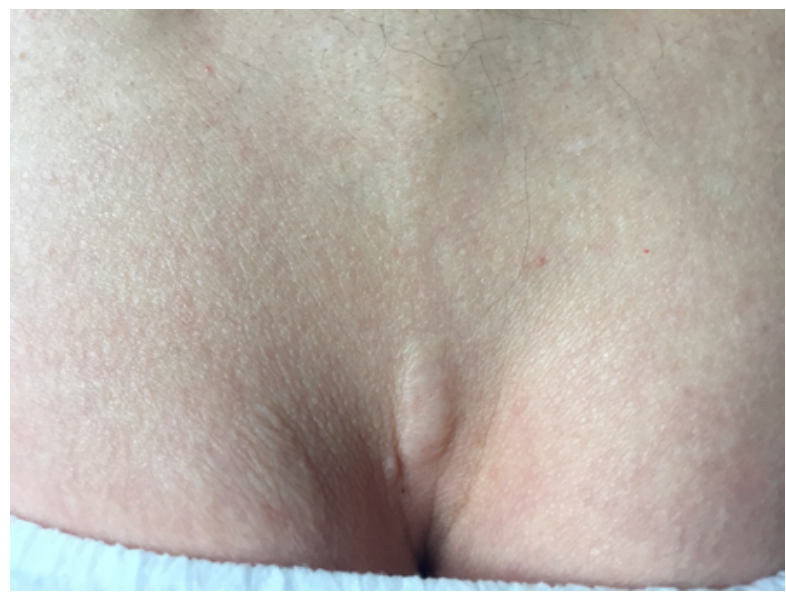


Fig. 3. Tomography scan of the brain with subordinate and supratentorial nodules

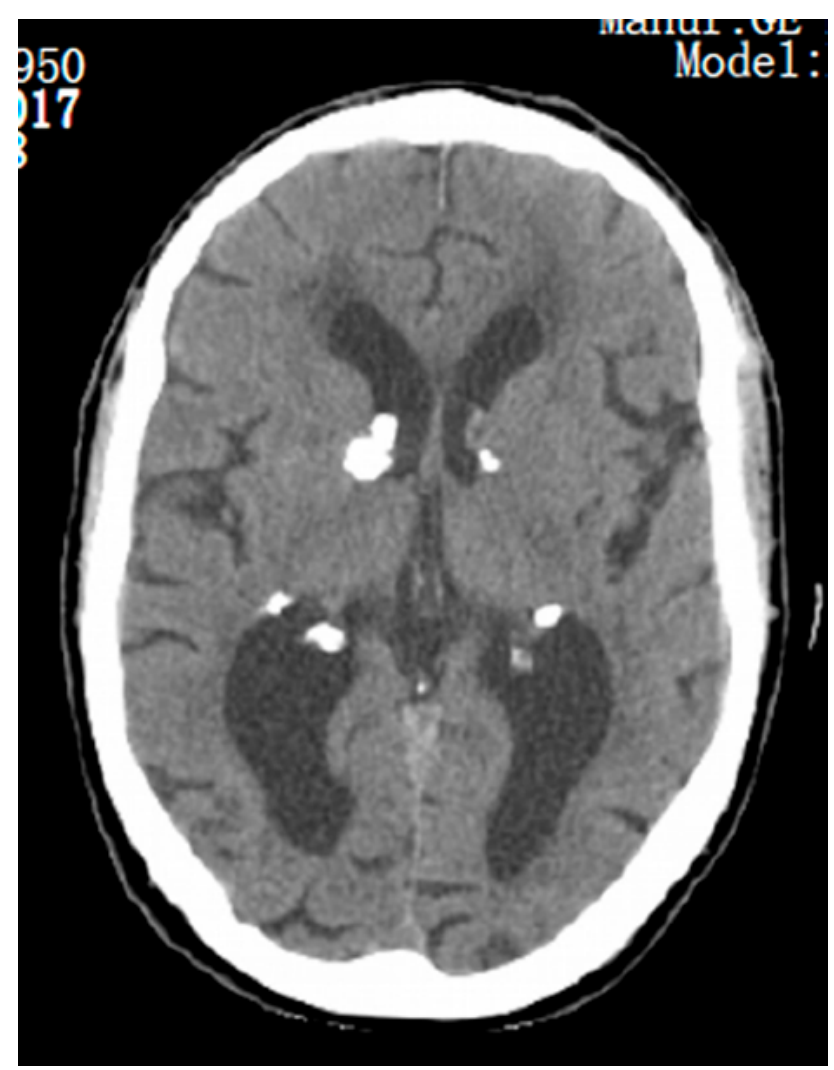

\section{Discussion}

Tuberous sclerosis complex (TSC) or Bourneville's disease was first described by Desiree-Magloire Bourneville in 1880². It is a rare genetic disorder of autosomal dominant inheritance with an incidence of approximately 1 in 5000 to 10,000 live births ${ }^{3}$. It has been determined that mutations in two genes are responsible for the development of tuberous sclerosis: TSC1 gene, called the hamartin gene, is located on chromosome 9 , and the TSC2 gene, called the tuberin gene, is located on chromosome 16. This hamartin--tuberin complex inhibits mTOR (mammalian target of rapamycin), a serine/threonine protein kinase that regulates cell growth and differentiation. When either of these tumor suppressor genes contains a defect, tumors in the form of normal organ tissues (hamartomas) can result4.

As an autosomal dominant disease, one--third of the cases derive from an altered TSC1 or TSC2 gene inheritance. A patient with TSC has a $50 \%$ chance of having a child affected by the disease, and the risk of a healthy couple, who had a child with TSC, to have another child with the disease is $2 \%$. Medical evaluation of healthy family members, who are planning to have children, should be performed by a dermatological examination, renal ultrasonography and cranial CT scan. Genetic evaluation by polymerase chain reaction, DNA sequencing and TSC1 and TSC2 dosage is not routinely indicated but helps when the clinical and radiological diagnosis is uncertain, especially in pre--natal cases ${ }^{4}$.

TSC is highly variable in its age of onset and severity, even within the same family. Its phenotypic expression involves many organ systems, including multiple benign hamartomas of the

Table 1. Diagnostic criteria for tuberous sclerosis complex. Definite diagnosis: 2 major features or one major feature with $\geq 2$ minor features. Possible diagnosis: either 1 major feature or $\geq 2$ minor features. ${ }^{*}$ A combination of the two major clinical features (lymphangioleiomyomatosis and angiomyolipomas) without other features doesn't meet criteria for definite diagnosis

\begin{tabular}{|c|c|c|}
\hline $\begin{array}{c}\text { Genetic diagnostic } \\
\text { criteria }\end{array}$ & \multicolumn{2}{|c|}{ Clinical diagnostic criteria } \\
\hline $\begin{array}{c}\text { Identification of } \\
\text { either TSC1 or TSC2 } \\
\text { pathogenic mutation } \\
\text { in DNA from normal } \\
\text { tissue }\end{array}$ & $\begin{array}{c}\text { Cortical displasias (includes tubers } \\
\text { and cerebral white matter radial } \\
\text { migration lines) }\end{array}$ & Dental enamel pits (>3) \\
\cline { 2 - 3 } & Subependymal nodules & Intraoral fibromas ( $\geq 2)$ \\
\cline { 2 - 3 } & Angiofibromas ( $\geq 3$ ) or fibrous \\
cephalic plaque & Retinal achromatic patch \\
\cline { 2 - 3 } & Ungual fibroma ( $\geq 2)$ & Confetti skin lesions \\
\cline { 2 - 3 } & Hypomelanotic macules ( $\geq 3$, at \\
least 5 mm in diameter) & Nonrenal hamartomas \\
\cline { 2 - 3 } & Shagreen patch & Multiple renal cysts \\
\cline { 2 - 3 } & Multiple retinal hamartomas & \\
\cline { 2 - 3 } & Cardiac rhabdomyoma & \\
\cline { 2 - 3 } & Angiomyolipomas ( $\geq 2)^{*}$ & \\
\cline { 2 - 3 } & Lymphangioleiomyomatosis* & \\
\hline
\end{tabular}


brain, eyes, heart, lung, liver, kidney, and skin that lead to organ dysfunction as the normal parenchyma is replaced by a variety of cell types ${ }^{5}$.

The diagnosis of TSC is made clinically. Diagnostic criteria for TSC is as given in the table 1. Definite diagnosis is made when two major or one major plus two minor features are present. Genetic testing is not required to make a diagnosis in patients who fulfill criteria for definite TSC, but it is helpful for family studies ${ }^{6}$. Nearly all patients with TSC have one or more of the skin lesions characteristic of the disorder (hypopigmented macules; angiofibromas; Shagreen patches). A distinctive brown fibrous plaque on the forehead may be the most readily recognized feature. International guidelines for TSC recommend performing a detailed skin examination at the time of diagnosis and annually thereafter. Although, there is no significant risk of malignant transformation, good sun protection, closer surveillance and intervention (laser therapy, dermabrasion) is recommended for skin lesions that rapidly change in size or number, and for those that cause pain, bleeding, functional impairment or social problems ${ }^{7}$.

The major neurological manifestations of TSC are seizures, autism, developmental delay and behavioral and psychiatric disorders. They usually associate with brain lesions including glioneuronal hamartomas (also called tubers), periventricular giant cell astrocytomas and abnormalities of cerebral white matter. Seizures are the most common and difficult aspect of management in TSC. The International Tuberous Sclerosis Complex Consensus Group recommends vigabatrin as first--line therapy for infantile spasms, and oxcarbazepine or carbamazepine for simple partial or complex partial. Approximately 63\% of patients with TSC and epilepsy develop medically intractable epilepsy and the treatment options for these patients include a ketogenic diet; vagus nerve stimulation; epilepsy surgery; and everolimus, a mTOR inhibitor. For brain tumors there are two main treatments: medical therapy with mTOR inhibitor or surgical resection. For the neuropsychiatric disorders, measures include early intervention and individual education programs, social support and psychiatric evaluation and treatment ${ }^{8}$.

Ophtalmologic involvement is described as the presence of more than one retinal hamartoma that have similar histologic features to the tubers located in the brain. Retinal achromatic patches can also occur 5 .

Renal lesions such as angiomyolipomas, cysts, lymphangiomas and renal cell carcinoma can occur and their prevalence increases with age. Nephron--saving surgery or partial nephrectomy can be performed when renal lesions are symptomatic. Total nephrectomy is reserved for cases with suspected malignancy and non--functioning kidneys with uncontrolled hypertension ${ }^{5}$.
Some adults with TSC develop pulmonary disease that is indistinguishable from the diffuse interstitial fibrosis known as lymphangioleiomyomatosis (LAM). This condition represents a cystic lung disease that can result in significant limitation in pulmonary function. The most common presenting features of LAM are dyspnea and pneumothorax. The treatment of LAM associated with tuberous sclerosis is identical to that of sporadic LAM. General measures include smoking cessation, bronchodilators, supplemental oxygen and pulmonary rehabilitation. Sirolimus and everolimus are first and second--line treatment, respectively, for patients with moderate--to--severe disease. For patients with advanced LAM or for those refractory to MTOR inhibitors, lung transplantation is a therapeutic option?.

Cardiac rhabdomyomas usually do not cause serious medical problems but, if symptomatic, surgical resection maybe necessary ${ }^{10}$.

In conclusion, tuberous sclerosis complex is highly variable and the majority of the cases are diagnosed during childhood. This report shows a delayed and opportunistic diagnosis, but as disease manifestations continue to develop over the lifetime of an affected individual, it is of the utmost importance the implementation of an appropriate medical surveillance and treatment 6 .

\section{References}

1 Schwartz RA, Fernandez G, Kotulska K, Jozwiak S. Tuberous sclerosis complex: advances in diagnosis, genetics, and management. J Am Acad Dermatol 2007; 57:189

2 Curatolo P, Maria BL. Tuberous sclerosis. In: Dulac 0, Lassonade M, Sarnat HB, editors. Pediatric Neurology part I. Elsevier B.V; 2013. P.323--31. (Handbooks of Clinical Neurology; volume 111)

3 Curatolo P, Bombardieri R, Jozwiak S. Tuberous sclerosis. Lancet 2008; 372:657 4 Van Slegtenhorst M, Nellist M, Nagelkerken B, Cheadle J, Snell R, van den Ouweland A, et al. Interaction between hamartin and tuberin, the TSC1 and TSC2 gene products. Hum Mol Genet 1998;7:1053--7

5 Northrup H, Koenig MK, Pearson DA, Au KS. Tuberous Sclerosis Complex. 1999 Jul 13 [Updated 2015 Sep 3]. In: Adam MP, Ardinger HH, Pagon RA, et al., editors. GeneReviews ${ }^{\circledR}$ [Internet]. Seattle (WA): University of Washington, Seattle; 1993-2017. Available from: https://www.ncbi.nlm.nih.gov/books/NBK1220/

6 Northrup H, Krueger DA. International Tuberous Sclerosis Complex Consensus Group. Tuberous sclerosis complex diagnostic criteria uptodate: frecommendations of the 2012 International Tuberous Sclerosis Complex Consensus Conference. Pediatr Neurol 2013; 49:243

7 Yates JR, Maclean C, Higgins JN, Humphrey A, le Marechal K, Clifford M, et al. The Tuberous Sclerosis 2000 Study: presentation, initial assessments and implications for diagnosis and management. Arch Dis Child 2011; 96:1020

8 Crino P, Mehta R, Vinters H. Pathogenesis of TSC in the brain. In: Kwiatkowsi D, Whittemore V, Thiele E, editors. Tuberous Sclerosis Complex: Genes, Clinical Features, and Therapeutics. Weinheim: Wiley--Blackwell; 2010. pp. 285-309.

9 von Ranke FM, Zanetti G, e Silva JL, et al. Tuberous Sclerosis Complex: State--of-the-Art Review with a Focus on Pulmonary Involvement. Lung 2015; 193:619.

10 Jozwiak S, Domanska--Pakiela D, Kwiatkowski DJ, Kotulska K. Multiple cardiac rhabdomyomas as a sole symptom of tuberous sclerosis complex: case report with molecular confirmation. J Child Neurol 2005; 20:988 